\title{
Large-sample inference in the general AR(1) model*
}

\author{
Efstathios Paparoditis \\ Dept. of Mathematics and Statistics \\ University of Cyprus, P.O. Box 537 \\ Nicosia, CYPRUS
}

\author{
Dimitris N. Politis \\ Department of Mathematics \\ University of California, San Diego \\ La Jolla, CA 92093-0112, USA
}

\begin{abstract}
The situation where the available data arise from a general $\operatorname{AR}(1)$ model is discussed, and two new avenues for constructing confidence intervals for the unknown autoregressive root are proposed, one based on a Central Limit Theorem, and the other based on the block-bootstrap. The two new methodologies rely on clever pre-processing of the original series, and are subsequently free of the difficulties present in previous methods that were due to data nonstationarity and/or discontinuity in the limit distribution in the case of a unit root. Some finitesample simulations are also presented supporting the applicability of the proposed methods, and the problem of bootstrap block size choice is discussed.
\end{abstract}

AMS 1980 subject classifications: primary 62M20; secondary $62 \mathrm{G} 05$.

Keywords and phrases: Bootstrap, confidence intervals, hypothesis tests, resampling, stationarity, unit root.

${ }^{*}$ Research partly supported by NSF Grant DMS-97-03964. 


\section{Introduction}

Consider time series data $\left\{X_{1}, \ldots, X_{n}\right\}$ arising from the familiar $\operatorname{AR}(1)$ model

$$
X_{t}=\mu+\rho X_{t-1}+\epsilon_{t}, \quad t=0, \pm 1, \pm 2, \ldots
$$

for some $\mu \in \mathbf{R}$, and $\rho \in[-1,1]$. The sequence $\left\{\epsilon_{t}\right\}$ is commonly termed the innovation' sequence; we will assume throughout the paper that:

$\left\{\epsilon_{t}\right\}$ is a strictly stationary, mean-zero, white noise (i.e., uncorrelated sequence) with $\operatorname{Var}_{t}=\sigma^{2} \in(0, \infty)$.

The three parameters $\mu, \rho$, and $\sigma^{2}$ are typically unknown and to be estimated from the observed data.

It is obvious that if $|\rho|<1$, the sequence $\left\{X_{t}\right\}$ is also strictly stationary; consequently, in this case we have available both nonparametric block-bootstrap methods (cf., Künsch (1989), Politis and Romano (1992a,b, 1994a)), as well as semiparametric bootstrap methods (in case the innovation sequence $\left\{\epsilon_{t}\right\}$ is actually i.i.d. - not just uncorrelated), that is, resampling of residuals as in Freedman (1984), Efron and Tibshirani (1986), Bose (1988). However, for $\rho=1$ the sequence $\left\{X_{t}\right\}$ is not stationary and the aforementioned methods break down; see e.g. Basawa et al. (1991). A similar breakdown occurs in the (admittedly less interesting) case where $\rho=-1$.

A good number of papers in the econometrics literature deal with model (1) under the assumption that $-1<\rho \leq 1$, i.e., the possibility of a unit root; see e.g. Hamilton (1994) or Fuller (1996) and the references therein. The traditional approach so far has been based on the Dickey and Fuller (1979) pioneering work and consists of conducting a test of the null hypothesis that there is a unit root, i.e., that $\rho=1$; in this connection see also Phillips and Perron (1988), and Ferretti and Romo (1996).

Recently, however, there has been some interest in the attempt to go beyond the simple unit root test; see e.g. Elliot and Stock (1994), or Stock (1991) who makes the strong point that "reporting only unit root tests and point estimates of the largest root 
is unsatisfying as a description of the data: this fails to convey information about the sampling uncertainty or, more precisely, the range of models (i.e., values of $\rho$ ) that are consistent with the observed data". Stock (1991) actually developed confidence intervals for $\rho$ based on 'local-to-unity' asymptotics; however, the finite-sample simulation studies in Stock (1991) show that his method seems to work well only if $\rho$ is very close to one. If $\rho \neq 1$, coverage probability of confidence intervals will gradually deteriorate as the sample size increases. Hansen (1997) proposed the 'grid-bootstrap' to address this situation, and reports improved performance. Datta and Sriram (1997) proposed a modified bootstrap in the same setting. Finally, Romano and Wolf (1998) applied the general subsampling methodology of Politis and Romano (1994b) to the AR(1) model with good results; see also Politis, Romano and Wolf (1999) for more details.

Nevertheless, it seems that if the objective is just to construct confidence intervals for $\rho$ in the $\operatorname{AR}(1)$ model, there exists an easy, natural and very simple way to look at the problem that avoids 'local-to-unity' asymptotics, and even by-passes the complications of the difficult (and discontinuous in $\rho$ ) limit distribution of $\rho$ 's estimator (cf. Fuller (1996)). This easy methodology is based on the age-old notion of pre-processing a time series and is the subject of the present paper.

In Section 2 we present the appropriate pre-processing methods for the AR(1) model with possible unit (or negative unit) root; we also derive confidence intervals for $\rho$ based on a Central Limit Theorem under the assumption that the innovation sequence $\left\{\epsilon_{t}\right\}$ is independent, identically distributed (i.i.d.). In Section 3 we review the blocks-of-blocks bootstrap methodology of Politis and Romano (1992a), we present a bootstrap consistency theorem under minimal assumptions, and we apply the blocksof-blocks bootstrap in the context of the AR(1) model with possible unit root. Section 4 contains some finite-sample simulations supporting the applicability of the proposed methods. The paper ends with a brief Section with our conclusions; as usual, all proofs are placed in the Appendix. 


\section{Confidence intervals in the general AR(1) model}

\subsection{The AR(1) model with possible unit root}

In this subsection we consider model (1) under the assumption that $-1<\rho \leq 1$, and construct the differenced series

$$
Y_{t} \equiv X_{t}-X_{t-1}, \quad t=0, \pm 1, \pm 2, \ldots
$$

The following lemma is the key result for our methodology; it presents the crucial observation that differencing is indeed the correct pre-processing tool in this case.

Lemma 1 For any value of $\rho \in(-1,1]$, the sequence $\left\{Y_{t}\right\}$ is strictly (and weakly) stationary, and its lag-1 autocorrelation coefficient is given by $\rho_{Y} \equiv \operatorname{Corr}\left(Y_{t}, Y_{t+1}\right)=$ $(\rho-1) / 2$.

An inspection of the proof of Lemma 1 yields the following interesting corollary.

Corollary 1 Under the constraint $-1<\rho \leq 1$, the lag-1 autocorrelation coefficient of the stationary sequence $\left\{Y_{t}\right\}$ satisfies $-1<\rho_{Y} \leq 0$, while its mean is given by:

$$
E Y_{t}= \begin{cases}0 & \text { if }-1<\rho<1 \\ \mu & \text { if } \rho=1\end{cases}
$$

If it so happens that the value of $\mu$ is known (and nonzero), or (more realistically) if it is known that $|\mu| \geq \varepsilon$, for some $\varepsilon>0$, then an easy (and powerful) test of the hypothesis $\rho=1$ vs. $-1<\rho<1$ can be performed via a test on the sample mean of the stationary sequence $\left\{Y_{t}\right\}$. Although we will not pursue this particular avenue any further, we will use (and elaborate upon) the notion of focusing on the new sequence $\left\{Y_{t}\right\}$ instead of the sequence $\left\{X_{t}\right\}$.

This general idea is based on Lemma 1 that affords us a new, nontrivial, and unifying perspective to the $\operatorname{AR}(1)$ problem: regardless of the value of $\rho$ (within the 
allowed limits $-1<\rho \leq 1$ ) we can now focus on a new, always stationary series whose lag-1 autocorrelation coefficient is related to $\rho$ by the one-to-one transformation given by $\rho_{Y}=(\rho-1) / 2$.

Consequently, under appropriate additional conditions, we can use either bootstrap or standard asymptotic methods on the stationary $\left\{Y_{t}\right\}$ sequence in order to derive confidence intervals for $\rho_{Y}$; the latter intervals can then be immediately worked out (i.e. 'solved') to yield confidence intervals for $\rho$. The extra conditions that are required have to do primarily with weak dependence properties of the $\left\{X_{t}\right\}$ and $\left\{\epsilon_{t}\right\}$ sequences; recall that the $\left\{\epsilon_{t}\right\}$ sequence was assumed uncorrelated but not necessarily independent. We elaborate on bootstrap issues in Section 3; in the meantime, we offer the following lemma that is of some interest in connection with the application of standard asymptotic methods.

Lemma 2 The stationary sequence $\left\{Y_{t}\right\}$ satisfies:

$$
Y_{t}= \begin{cases}\epsilon_{t}+(\rho-1)\left(\epsilon_{t-1}+\rho \epsilon_{t-2}+\rho^{2} \epsilon_{t-3}+\cdots\right) & \text { if }-1<\rho<1 \\ \mu+\epsilon_{t} & \text { if } \rho=1,\end{cases}
$$

where the infinite sum of random variables converges almost surely and in mean-square.

Note that for all values of $\rho \in(-1,1],\left\{Y_{t}\right\}$ is a one-sided $\mathrm{MA}(\infty)$ model (with geometrically decreasing coefficients) — see e.g. Priestley (1981). In addition, if the sequence $\left\{\epsilon_{t}\right\}$ is actually i.i.d. and not just uncorrelated, $\left\{Y_{t}\right\}$ is a linear time series. But, in this case, there exists a Central Limit Theorem for the lag-1 sample autocorrelation given by:

$$
\hat{\rho}_{Y}=\frac{\sum_{t=2}^{n-1}\left(Y_{t}-\bar{Y}\right)\left(Y_{t+1}-\bar{Y}\right)}{\sum_{t=2}^{n}\left(Y_{t}-\bar{Y}\right)^{2}},
$$

where $\bar{Y}=(n-1)^{-1} \sum_{t=2}^{n} Y_{t}$; recall that from the data $\left\{X_{1}, \ldots, X_{n}\right\}$ we can only construct the stretch $\left\{Y_{2}, \ldots, Y_{n}\right\}$ of the $\left\{Y_{t}\right\}$ sequence. The following theorem confirms these ideas. 
Theorem 1 Assume model (1) under the constraint $-1<\rho \leq 1$, and with the sequence $\left\{\epsilon_{t}\right\}$ being i.i.d. Then we have

$$
\sqrt{n-1}\left(\hat{\rho}_{Y}-\rho_{Y}\right) \stackrel{\mathcal{L}}{\Longrightarrow} N\left(0, \tau_{Y}^{2}\right)
$$

as $n \rightarrow \infty$, where $\tau_{Y}^{2}=(\rho+1) / 2$.

Note that to actually use the Central Limit Theorem of Theorem 1 to set confidence intervals for $\rho_{Y}$ of the type

$$
\left[\hat{\rho}_{Y}-\frac{1.645 \tau_{Y}}{\sqrt{n-1}}, \hat{\rho}_{Y}+\frac{1.645 \tau_{Y}}{\sqrt{n-1}}\right]
$$

(for $90 \%$ confidence level, say) we require a consistent estimate $\hat{\tau}_{Y}^{2}$ of $\tau_{Y}^{2}$. This is easily accomplished by plugging in the natural (and consistent) estimator $\hat{\rho}=2 \hat{\rho}_{Y}+1$ of $\rho$ in the formula for $\tau_{Y}^{2}$, i.e., letting

$$
\hat{\tau}_{Y}^{2}=(\hat{\rho}+1) / 2=\hat{\rho}_{Y}+1
$$

Finally, a confidence interval (of approximate $90 \%$ coverage level) for $\rho_{Y}$ is given by

$$
\left[\hat{\rho}_{Y}-\frac{1.645 \hat{\tau}_{Y}}{\sqrt{n-1}}, \hat{\rho}_{Y}+\frac{1.645 \hat{\tau}_{Y}}{\sqrt{n-1}}\right]
$$

which can be 'solved' to give a confidence interval (of approximate $90 \%$ coverage level) for $\rho$ of the form

$$
\left[1+2\left(\hat{\rho}_{Y}-\frac{1.645 \hat{\tau}_{Y}}{\sqrt{n-1}}\right), 1+2\left(\hat{\rho}_{Y}+\frac{1.645 \hat{\tau}_{Y}}{\sqrt{n-1}}\right)\right]
$$

Confidence interval (4) is remarkably quick-and-easy in its application. Nevertheless, there are two important reasons for which we might consider constructing bootstrap confidence intervals for $\rho_{Y}$ (and subsequently for $\rho$ as well) instead of the confidence intervals based on the Central Limit Theorem that are given above.

1. The Central Limit Theorem of Theorem 1 -as well as that of Theorem 7.2.2 in Brockwell and Davis (1991) - are valid only under the assumption that the innovation sequence $\left\{\epsilon_{t}\right\}$ is actually i.i.d. and not just uncorrelated. If the i.i.d. assumption breaks down, then the intervals (3) and (4) can give erroneous information; see Romano and Thombs (1996) for more details on this observation. 
2. In numerous situations so far (see e.g. Hall (1992), Shao and Tu (1995), Efron and Tibshirani (1993), Davison and Hinkley (1997)) the bootstrap has been shown to outperform the normal approximation when they are both applicable.

Thus, the bootstrap may be recommendable both in the context of robustness against possible dependence in the innovation sequence, and/or for potentially increasing performance. Of course, this comes at a price: the bootstrap is quite computationally intensive while the interval (4) can even be calculated by hand. In addition, for the implementation of nonparametric block-bootstrap methods, the user has to specify the block size to be used which is not trivial; misspecification of the block size in practice may well destroy any improvement in performance - see our Section 4 for more discussion on this important issue.

The block-bootstrap (and in particular, the so-called blocks-of-blocks bootstrap) for $\rho_{Y}$ is the subject of Section 3 in which the assumption that the innovation sequence $\left\{\epsilon_{t}\right\}$ is i.i.d. is replaced by a mixing assumption. Nevertheless, before going into that, let us consider a quick-and-easy way of improving upon interval (4) in the spirit of R.A. Fisher.

Recall that, in the case of estimation of a correlation coefficient under the set-up of i.i.d. observations from a bivariate normal distribution, Fisher's $z$ transformation is recommended as a normalizing and variance stabilizing transformation; see e.g. Efron and Tibshirani (1993). Our set-up here is quite different but we can still find a variance stabilizing transformation based on our Theorem 1: it is given by the function $\eta(x)=\sqrt{1+x}$. Corollary 2 follows immediately by an application of the so-called $\delta$ method (i.e., a Taylor expansion).

Corollary 2 Assume model (1) under the constraint $-1<\rho \leq 1$, and with the sequence $\left\{\epsilon_{t}\right\}$ being i.i.d. Then we have

$$
\sqrt{n-1}\left(\eta\left(\hat{\rho}_{Y}\right)-\eta\left(\rho_{Y}\right)\right) \stackrel{\mathcal{L}}{\Longrightarrow} N(0,1 / 4)
$$

as $n \rightarrow \infty$. 
Based on Corollary 2 we construct a new confidence interval (of approximate $90 \%$ coverage level) for $\rho_{Y}$ by

$$
\left[-1+\left(\sqrt{\hat{\rho}_{Y}+1}-\frac{1.645}{2 \sqrt{n-1}}\right)^{2},-1+\left(\sqrt{\hat{\rho}_{Y}+1}+\frac{1.645}{2 \sqrt{n-1}}\right)^{2}\right]
$$

which can again be 'solved' to give a confidence interval (of approximate $90 \%$ coverage level) for $\rho$ of the form

$$
\left[-1+2\left(\sqrt{\hat{\rho}_{Y}+1}-\frac{1.645}{2 \sqrt{n-1}}\right)^{2},-1+2\left(\sqrt{\hat{\rho}_{Y}+1}+\frac{1.645}{2 \sqrt{n-1}}\right)^{2}\right] .
$$

Confidence interval (6) is expected to be more accurate than confidence interval (4).

\subsection{The AR(1) model with possible negative unit root}

For completeness, we briefly discuss the case where $\rho$ might take the value -1 . In this connection, we consider model (1) under the assumption that $-1 \leq \rho<1$, and construct the integrated series

$$
W_{t} \equiv X_{t}+X_{t-1}, \quad t=0, \pm 1, \pm 2, \ldots
$$

That this forms the correct pre-processing tool in this case is confirmed by the following lemma whose proof is similar to the proof of Lemma 1, and thus ommited.

Lemma 3 For any value of $\rho \in[-1,1)$, the sequence $\left\{W_{t}\right\}$ is strictly (and weakly) stationary, and its lag-1 autocorrelation coefficient is given by $\rho_{W} \equiv \operatorname{Corr}\left(W_{t}, W_{t+1}\right)=$ $(\rho+1) / 2$.

We also have analogs of Corollary 1 and Lemma 2.

Corollary 3 Under the constraint $-1 \leq \rho<1$, the lag-1 autocorrelation coefficient of the stationary sequence $\left\{W_{t}\right\}$ satisfies $0 \leq \rho_{W}<1$, while its mean is given by:

$$
E W_{t}= \begin{cases}0 & \text { if }-1<\rho<1 \\ \mu & \text { if } \rho=-1\end{cases}
$$


Lemma 4 The stationary sequence $\left\{W_{t}\right\}$ satisfies:

$$
W_{t}= \begin{cases}\epsilon_{t}+(\rho+1)\left(\epsilon_{t-1}+\rho \epsilon_{t-2}+\rho^{2} \epsilon_{t-3}+\cdots\right) & \text { if }-1<\rho<1 \\ \mu+\epsilon_{t} & \text { if } \rho=-1,\end{cases}
$$

where the infinite sum of random variables converges almost surely and in mean-square.

As before, there exists a Central Limit Theorem for the lag-1 sample autocorrelation given by:

$$
\hat{\rho}_{W}=\frac{\sum_{t=2}^{n-1}\left(W_{t}-\bar{W}\right)\left(W_{t+1}-\bar{W}\right)}{\sum_{t=2}^{n}\left(W_{t}-\bar{W}\right)^{2}},
$$

where $\bar{W}=(n-1)^{-1} \sum_{t=2}^{n} W_{t}$; recall that from the data $\left\{X_{1}, \ldots, X_{n}\right\}$ we can only construct the stretch $\left\{W_{2}, \ldots, W_{n}\right\}$ of the $\left\{W_{t}\right\}$ sequence.

Theorem 2 Assume model (1) under the constraint $-1 \leq \rho<1$, and with the sequence $\left\{\epsilon_{t}\right\}$ being i.i.d. Then we have

$$
\sqrt{n-1}\left(\hat{\rho}_{W}-\rho_{W}\right) \stackrel{\mathcal{L}}{\Longrightarrow} N\left(0, \tau_{W}^{2}\right)
$$

as $n \rightarrow \infty$, where $\tau_{W}^{2}=(1-\rho) / 2$.

In order to use the Central Limit Theorem of Theorem 2 to set confidence intervals for $\rho_{W}$ of the type

$$
\left[\hat{\rho}_{W}-\frac{1.645 \tau_{W}}{\sqrt{n-1}}, \hat{\rho}_{W}+\frac{1.645 \tau_{W}}{\sqrt{n-1}}\right]
$$

(for $90 \%$ confidence level, say) we require a consistent estimate $\hat{\tau}_{W}^{2}$ of $\tau_{W}^{2}$. This is accomplished by plugging in the consistent estimator $\hat{\rho}=2 \hat{\rho}_{W}-1$ of $\rho$ in the formula for $\tau_{W}^{2}$, i.e., letting

$$
\hat{\tau}_{W}^{2}=(1-\hat{\rho}) / 2=1-\hat{\rho}_{W} .
$$

Alternatively, we can use a variance stabilization transformation similar to Corollary 2. Our Theorem 2 suggests that such a transformation is now given by the function $\lambda(x)=\sqrt{1-x}$. Corollary 4 follows immediately by an application of the $\delta$-method. 
Corollary 4 Assume model (1) under the constraint $-1 \leq \rho<1$, and with the sequence $\left\{\epsilon_{t}\right\}$ being i.i.d. Then we have

$$
\sqrt{n-1}\left(\lambda\left(\hat{\rho}_{Y}\right)-\lambda\left(\rho_{Y}\right)\right) \stackrel{\mathcal{L}}{\Longrightarrow} N(0,1 / 4)
$$

as $n \rightarrow \infty$.

\section{Blocks-of-blocks bootstrap for the lag-1 autocorrelation}

\subsection{Case of a general stationary series}

As previously mentioned, if the innovation sequence $\left\{\epsilon_{t}\right\}$ can not be assumed to be i.i.d., then our only option is to resort to bootstrapping; the assumption that $\left\{\epsilon_{t}\right\}$ is i.i.d. can then be replaced by an appropriate mixing condition. In what follows, we will focus on the so-called blocks-of-blocks bootstrap because in a recent comparative study (cf. Table 8.2 in Davison and Hinkley (1997)) the blocks-of-blocks bootstrap seemed to be the best performing among different block-bootstrap methods for the autocorrelation of a stationary series. The blocks-of-blocks bootstrap was proposed by Politis and Romano (1992a) as a generalization of Künsch's (1989) block-bootstrap; its implementation on the lag-1 autocorrelation is reviewed below. For reasons to be explained in the sequel, the circular 'wrap-around' proposed in Politis and Romano (1992b) is also employed.

Suppose $\left\{U_{1}, \ldots, U_{N}\right\}$ is an observed stretch from a strictly stationary series $\left\{U_{t}\right\}$ with autocorrelation $\rho_{U}(k)=\operatorname{Corr}\left(U_{1}, U_{1+k}\right)$. As before, let $\rho_{U}=\rho_{U}(1)$ that is assumed to lie in the interval $(-1,1)$; the issue is to construct confidence intervals for $\rho_{U}$. 
The time series $\left\{U_{t}\right\}$ will be assumed to have a weak dependence structure. Specifically, consider the strong mixing coefficients $\alpha_{U}(k)=\sup _{A, B}|P(A \cap B)-P(A) P(B)|$, where $A \in \mathcal{F}_{-\infty}^{0}, B \in \mathcal{F}_{k}^{\infty}$ are events in the $\sigma$-algebras generated by $\left\{U_{t}, t \leq 0\right\}$ and $\left\{U_{t}, t \geq k\right\}$ respectively. A mixing condition will be of the type $\alpha_{U}(k) \rightarrow 0$, as $k \rightarrow \infty$, where this convergence happens at a fast enough, problem-dependent rate.

The Blocks-of-Blocks Circular Bootstrap for the Lag-1 AutocorreLATION.

1. Construct a stretch of the new vector series $\left\{V_{t}\right\}$ by defining

$$
V_{t}=\left(U_{t}, U_{t}^{2}, U_{t} U_{t+1}\right)^{\prime}, \quad t=1, \ldots, N-1
$$

2. Define the new multivariate statistic $\tilde{T}=(N-1)^{-1} \sum_{t=1}^{N-1} V_{t}$; denote $\tilde{T}^{(i)}$ the $i$ th coordinate of $\tilde{T}$. Observe that we can form (a version of) the lag-1 autocorrelation estimator by

$$
\tilde{\rho}_{U}=\frac{\tilde{T}^{(3)}-\left(\tilde{T}^{(1)}\right)^{2}}{\tilde{T}^{(2)}-\left(\tilde{T}^{(1)}\right)^{2}} .
$$

3. Now perform the circular bootstrap of Politis and Romano (1992b) on the new series $\left\{V_{t}\right\}$. To begin with, consider a 'circular' extension of $V_{t}$ for all $t$-values by defining

$$
V_{t}=V_{t_{\text {odulo }(N-1)}}
$$

4. Pick an integer block size $b(<N-1)$, and define $\mathcal{B}_{j, b}$ to be the block of $b$ consecutive $V_{t}$ 's starting from $V_{j}$, that is, let $\mathcal{B}_{j, b}=\left(V_{j}, \ldots, V_{j-1+b}\right)$ for $j=$ $1, \ldots, N-1$. Sampling with replacement from the set $\left\{\mathcal{B}_{1, b}, \ldots, \mathcal{B}_{N-1, b}\right\}$ defines (conditionally on the original observations $V_{1}, \ldots, V_{N-1}$ ) a bootstrap probability measure denoted by $P^{*}$. Now let $Z_{1}, \ldots, Z_{k}$ be i.i.d. samples from $P^{*}$, where $k=[N / b]$ and $[\cdot]$ represents the integer part. Obviously, each $Z_{i}$ is a block of size $b$ which we denote as $Z_{i}=\left(z_{i 1}, \ldots, z_{i b}\right)$.

5. Concatenate the $z_{i j}$ in one long vector of size $L=k b$ denoted by $V_{1}^{*}, \ldots, V_{L}^{*}$, where $V_{i}^{*}=z_{r v}$, for $r=[i / b], v=i-b r$. Let $\tilde{T}^{*}=L^{-1} \sum_{t=1}^{L} V_{t}^{*}$, where $\tilde{T}^{*(i)}$ the 
$i$ th coordinate of $\tilde{T}^{*}$, and

$$
\tilde{\rho}_{U}^{*}=\frac{\tilde{T}^{*(3)}-\left(\tilde{T}^{*(1)}\right)^{2}}{\tilde{T}^{*(2)}-\left(\tilde{T}^{*(1)}\right)^{2}} .
$$

6. Finally, the circular blocks-of-blocks bootstrap estimate of $P\left\{\sqrt{N-1}\left(\tilde{\rho}_{U}-\rho_{U}\right) \leq\right.$ $x\}$ is given by $P^{*}\left\{\sqrt{L}\left(\tilde{\rho}_{U}^{*}-\tilde{\rho}_{U}\right) \leq x\right\}$, where $x$ is any real number. For practical implementation, $P^{*}$ probabilities are actually computed by Monte-Carlo simulation.

7. Motivated by Corollaries 2 and 4, recall that the bootstrap also benefits from a normalizing and variance stabilizing transformation when such a transformation exists; cf. Efron and Tibshirani (1993). Thus, suppose that such a transformation is given by some function $\zeta(x)$; then, the previously quoted bootstrap approximation

$$
P^{*}\left\{\sqrt{L}\left(\tilde{\rho}_{U}^{*}-\tilde{\rho}_{U}\right) \leq x\right\} \simeq P\left\{\sqrt{N-1}\left(\tilde{\rho}_{U}-\rho_{U}\right) \leq x\right\}
$$

implies (via the $\delta$-method) the following:

$$
P^{*}\left\{\sqrt{L}\left(\zeta\left(\tilde{\rho}_{U}^{*}\right)-\zeta\left(\tilde{\rho}_{U}\right)\right) \leq x\right\} \simeq P\left\{\sqrt{N-1}\left(\zeta\left(\tilde{\rho}_{U}\right)-\zeta\left(\rho_{U}\right)\right) \leq x\right\}
$$

If indeed $\zeta\left(\tilde{\rho}_{U}\right)$ has stabilized variance, i.e., if $\operatorname{Var}\left(\zeta\left(\tilde{\rho}_{U}\right)\right)$ does not depend on the unknown $\rho_{U}$, then approximation (9) will typically more accurate than approximation (8).

Observe that, by construction, $L /(N-1) \rightarrow 1$ as $N \rightarrow \infty$. Thus, for really large $N$, approximation (8) implies that for all real $x$,

$$
P^{*}\left\{\sqrt{N-1}\left(\tilde{\rho}_{U}^{*}-\tilde{\rho}_{U}\right) \leq x\right\} \simeq P\left\{\sqrt{N-1}\left(\tilde{\rho}_{U}-\rho_{U}\right) \leq x\right\}
$$

which can be interpreted as saying that the bootstrap quantiles of the distribution of $\tilde{\rho}_{U}^{*}$ approximate the quantiles of the true distribution of $\tilde{\rho}_{U}$; hence, the construction of bootstrap confidence intervals for $\rho_{U}$ is immediate. Similarly, bootstrap confidence intervals for $\zeta\left(\rho_{U}\right)$ can be gotten from equation (9) with the substitution $L \sim N-1$; 
the confidence intervals for $\zeta\left(\rho_{U}\right)$ can then be 'solved' to yield confidence intervals for $\rho_{U}$.

In the case of moderately large $N$, it is more accurate to take into account that $L$ and $N-1$ are not identical with an appropriate re-weighting of the bootstrap quantiles before they serve as approximations to the true quantiles; for more details, see the books by Hall (1992), Shao and Tu (1995), Efron and Tibshirani (1993), Davison and Hinkley (1997).

The following theorem quantifies conditions for the validity of asymptotic approximations (8) and (9). It is a corollary of the general consistency theorems in Politis and Romano (1992a,b) in conjunction with a more recent result of Radulovic (1996) that allows us to use less stringent moment and mixing conditions.

Theorem 3 Suppose that $\left\{U_{t}\right\}$ is a strictly stationary sequence such that $E\left|U_{t}\right|^{4+\delta}<$ $\infty$, and $\sum_{k=1}^{\infty} \alpha_{U}^{\delta /(2+\delta)}(k)<\infty$, for some constant $\delta>0$.

Let $b \rightarrow \infty$ but with $b / N \rightarrow 0$ as $N \rightarrow \infty$; then

$$
d\left(P^{*}\left\{\sqrt{L}\left(\tilde{\rho}_{U}^{*}-\tilde{\rho}_{U}\right) \leq \cdot\right\}, P\left\{\sqrt{N-1}\left(\tilde{\rho}_{U}-\rho_{U}\right) \leq \cdot\right\}\right) \stackrel{P}{\rightarrow} 0
$$

and

$$
d\left(P^{*}\left\{\sqrt{L}\left(\zeta\left(\tilde{\rho}_{U}^{*}\right)-\zeta\left(\tilde{\rho}_{U}\right)\right) \leq \cdot\right\}, P\left\{\sqrt{N-1}\left(\zeta\left(\tilde{\rho}_{U}\right)-\zeta\left(\rho_{U}\right)\right) \leq \cdot\right\}\right) \stackrel{P}{\rightarrow} 0
$$

where $d(\cdot, \cdot)$ is any metric metrizing weak convergence (e.g. Prohorov's metric).

If in addition, we have

$$
\lim _{N \rightarrow \infty} \operatorname{Var}\left(\sqrt{N-1} \tilde{\rho}_{U}\right)>0
$$

then the uniform metric can be used, i.e.,

$$
\sup _{x}\left|P^{*}\left\{\sqrt{L}\left(\tilde{\rho}_{U}^{*}-\tilde{\rho}_{U}\right) \leq x\right\}-P\left\{\sqrt{N-1}\left(\tilde{\rho}_{U}-\rho_{U}\right) \leq x\right\}\right| \stackrel{P}{\rightarrow} 0,
$$

and

$$
\sup _{x}\left|P^{*}\left\{\sqrt{L}\left(\zeta\left(\tilde{\rho}_{U}^{*}\right)-\zeta\left(\tilde{\rho}_{U}\right)\right) \leq x\right\}-P\left\{\sqrt{N-1}\left(\zeta\left(\tilde{\rho}_{U}\right)-\zeta\left(\rho_{U}\right)\right) \leq x\right\}\right| \stackrel{P}{\rightarrow} 0 .
$$


As previously discussed, approximations (14) and (15) are the ones used in practice for bootstrap confidence interval construction, and not (11) and (12). The reason is that the case where equation (13) does not hold is a trivial case in which confidence intervals are superfluous - the large-sample variability of the estimator being zero. Happily, it is very easy to empirically verify whether equation (13) does or does not hold.

Note that there is a wide variety of block size choices that will satisfy our theorem's requirements $b \rightarrow \infty$ with $b / N \rightarrow 0$ as $N \rightarrow \infty$. Nevertheless, for practical (finite-sample) implementation, a single block size must be chosen, and the quality of the bootstrap distribution approximation may crucially depend on the block size choice. In the context of our Theorem 3, i.e., for estimation of a (one-sided) distribution function, Hall et al. (1996) show that choosing the block size proportional to $N^{1 / 4}$ is asymptotically optimal; nevertheless, the optimal proportionality constant is highly problem-dependent and typically difficult to pin-point — see the discussion in our Section 4.

Finally, note that the main reason for using the 'circular' extension is that it affords us correct centering of the bootstrap distribution automatically. Without the 'circular' extension, equation (11) will not hold for all values of $b$ satisfying $b \rightarrow \infty$ and $b / N \rightarrow 0$ as $N \rightarrow \infty$ as given in Theorem 3. To obtain a correct approximation in the non-circular case, it would be necessary to use the artificially centered bootstrap distribution $P^{*}\left\{\sqrt{L}\left(\tilde{\rho}_{U}^{*}-E^{*} \tilde{\rho}_{U}^{*}\right) \leq x\right\}$ instead of the more natural $P^{*}\left\{\sqrt{L}\left(\tilde{\rho}_{U}^{*}-\tilde{\rho}_{U}\right) \leq x\right\}$ in equations (8) and (11) — with corresponding changes in equations (9) and (12); here, $E^{*}$ denotes expectation under the $P^{*}$ probability and would have to be computed by a separate computational or Monte-Carlo step. 


\subsection{Application to the case of a general AR(1) model}

We now assume the set-up of Section 2.1, i.e., model (1) for the $\left\{X_{t}\right\}$ series under the assumption that $-1<\rho \leq 1$, and focus on the case where $\left\{U_{t}\right\}$ is the stationary series $\left\{Y_{t}\right\}$, where $Y_{t} \equiv X_{t}-X_{t-1}, \quad t=0, \pm 1, \pm 2, \ldots$; the observed sample sizes are related by $N=n-1$.

Corollary 5 Assume that the data $\left\{X_{1}, \ldots, X_{n}\right\}$ were generated by model (1) under the assumption that $-1<\rho \leq 1$. Also assume that, for some constant $\delta>0$, $E\left|\epsilon_{t}\right|^{4+\delta}<\infty$, and $\sum_{k=1}^{\infty} \alpha_{Y}^{\delta /(2+\delta)}(k)<\infty$. Let $b \rightarrow \infty$ but with $b / n \rightarrow 0$ as $n \rightarrow \infty$; then equations (11) and (12) hold true with the series $\left\{Y_{t}\right\}$ in place of $\left\{U_{t}\right\}$, and $n-1$ in place of $N$. If in addition, $\lim _{n \rightarrow \infty} \operatorname{Var}\left(\sqrt{n-2} \tilde{\rho}_{Y}\right)>0$, then equations (14) and (15) hold true as well (with $\left\{Y_{t}\right\}$ in place of $\left\{U_{t}\right\}$, and $n-1$ in place of $N$ ).

The following remarks are in order.

- Note that the compact assumption $\sum_{k=1}^{\infty} \alpha_{Y}^{\delta /(2+\delta)}(k)<\infty$ contains in it the two possibilities: (a) that $\left\{X_{t}\right\}$ is mixing -in the case $-1<\rho<1$, and (b) that $\left\{X_{t}\right\}$ is a random walk with mixing innovations -in the case $\rho=1$.

- The assumptions of Corollary 5 are just strong enough to imply the asymptotically normality of $\hat{\rho}_{Y}$; however, the asymptotic variance of $\hat{\rho}_{Y}$ can not in general be calculated unless we assume that the innovation sequence $\left\{\epsilon_{t}\right\}$ is i.i.d. (or at least a martingale difference) in which case we would be falling back in a context similar to that of our Theorem 1.

- Observe that although $\sum_{k=1}^{\infty} \alpha_{Y}^{\delta /(2+\delta)}(k)<\infty$ implies $\sum_{k=1}^{\infty} \alpha_{\epsilon}^{\delta /(2+\delta)}(k)<\infty$, the converse is in general not true; see e.g. Andrews (1984). Nevertheless, in the set-up of Theorem 1 (where the innovations $\left\{\epsilon_{t}\right\}$ are assumed i.i.d.), the mixing condition $\sum_{k=1}^{\infty} \alpha_{Y}^{\delta /(2+\delta)}(k)<\infty$ follows immediately under the extra assumption that the innovations $\left\{\epsilon_{t}\right\}$ have an absolutely continuous distribution; see Doukhan (1994). Thus, in the set-up of Theorem 1, under the extra assumption that the 
innovations $\left\{\epsilon_{t}\right\}$ have an absolutely continuous distribution with a finite $(4+\delta)$ moment, we have concurrently available the confidence intervals (4) and (6), as well as the one obtained via the blocks-of-blocks bootstrap of Corollary 5 .

A similar result holds under the set-up of Section 2.2, i.e., model (1) for the $\left\{X_{t}\right\}$ series under the assumption that $-1 \leq \rho<1$. Thus, consider the case where $\left\{U_{t}\right\}$ is the stationary series $\left\{W_{t}\right\}$, where $W_{t} \equiv X_{t}+X_{t-1}, \quad t=0, \pm 1, \pm 2, \ldots$; the observed sample sizes are again related by $N=n-1$.

Corollary 6 Assume that the data $\left\{X_{1}, \ldots, X_{n}\right\}$ were generated by model (1) under the assumption that $-1 \leq \rho<1$. Also assume that, for some constant $\delta>0$, $E\left|\epsilon_{t}\right|^{4+\delta}<\infty$, and $\sum_{k=1}^{\infty} \alpha_{W}^{\delta /(2+\delta)}(k)<\infty$. Let $b \rightarrow \infty$ but with $b / n \rightarrow 0$ as $n \rightarrow \infty$; then equations (11) and (12) hold true with the series $\left\{W_{t}\right\}$ in place of $\left\{U_{t}\right\}$, and $n-1$ in place of $N$. If in addition, $\lim _{n \rightarrow \infty} \operatorname{Var}\left(\sqrt{n-2} \tilde{\rho}_{W}\right)>0$, then equations (14) and (15) hold true as well (with $\left\{W_{t}\right\}$ in place of $\left\{U_{t}\right\}$, and $n-1$ in place of $N$ ).

\section{Finite-sample simulations}

For the simulations, a number of stretches of the type $\left\{X_{1}, \ldots, X_{n}\right\}$ were generated from the $\operatorname{AR}(1)$ model (1) with $\mu=0$. We used three different sample sizes $n=60,120$ and 240, and three different values for $\rho=0.6,0.9$ and 1 ; these are the same choices used in the simulations of Hansen (1997).

We used two different choices for the innovation sequence $\left\{\epsilon_{t}\right\}$.

I.I.D. GAUSSIAN CASE : $\epsilon_{t}=z_{t}, t=0, \pm 1, \pm 2, \ldots$, where $\left\{z_{t}\right\}$ is i.i.d. $\mathrm{N}(0,1)$.

NON-GAUSSIAN (ARCH) CASE : $\epsilon_{t}=z_{t} z_{t-1}^{2}, t=0, \pm 1, \pm 2, \ldots$, where $\left\{z_{t}\right\}$ is i.i.d. $\mathrm{N}(0,1)$ as before; here $\left\{\epsilon_{t}\right\}$ follows a simple ARCH model, and is not indepen- 
dent — it is not even a martingale difference.

In each of the two above cases, we constructed equal-tailed confidence intervals for $\rho$ with nominal confidence level $90 \%$ based on:

1. Central Limit Theorem (CLT) -see eq. (4).

2. Central Limit Theorem with the use of transformation $\eta(\mathrm{CLT}-\eta)$-see eq. (6).

3. Blocks-of-blocks bootstrap (BBB) -see eq. (8).

4. Blocks-of-blocks bootstrap with the use of transformation $\eta(\mathrm{BBB}-\eta)$-see eq. (9) with $\zeta=\eta$.

As previously mentioned, Hall et al. (1996) showed that the optimal block size for the bootstrap is asymptotically proportional to $n^{1 / 4}$, i.e.,

$$
b_{o p t} \sim c_{X} n^{1 / 4}
$$

where the proportionality constant $c_{X}$ is generally intractable as it depends in an unknown way on features of the $X$-process.

The empirically observed coverage levels for the constructed confidence intervals are given in Tables 1 and 2. To compute the entries of Tables 1 and 2 we used the simple block size choice $b \sim n^{1 / 4}$. As a matter of fact, since $b$ must be an integer, we used the choice $b=\left[n^{1 / 4}+0.1\right]$, where [·] is the integer part; thus, we chose $b=2,3$, and 4 for $n=60,120$ and 240 respectively. The standard error of each of these empirical probabilities is 0.015 as each is based on 400 replications. Finally note that the bootstrap Monte-Carlo approximations involved the generation of 250 bootstrap 'pseudo'-series for each original series. 


\begin{tabular}{|c|c|c|c|c|c|c|c|c|c|}
\hline$n$ & \multicolumn{3}{|c|}{60} & \multicolumn{3}{c|}{120} & \multicolumn{3}{c|}{240} \\
\hline$\rho$ & 0.6 & 0.9 & 1.0 & 0.6 & 0.9 & 1.0 & 0.6 & 0.9 & 1.0 \\
\hline $\mathrm{CLT}$ & 0.8800 & 0.8875 & 0.8725 & 0.9000 & 0.9025 & 0.9100 & 0.9075 & 0.9150 & 0.8875 \\
\hline $\mathrm{CLT}-\eta$ & 0.8775 & 0.9000 & 0.8725 & 0.9050 & 0.9100 & 0.9075 & 0.9100 & 0.9225 & 0.9000 \\
\hline $\mathrm{BBB}$ & 0.8550 & 0.8725 & 0.8550 & 0.8750 & 0.8675 & 0.8775 & 0.8800 & 0.8825 & 0.8725 \\
\hline $\mathrm{BBB}-\eta$ & 0.8525 & 0.8775 & 0.8675 & 0.8725 & 0.8800 & 0.8925 & 0.8825 & 0.9000 & 0.8750 \\
\hline
\end{tabular}

Table 1: (GAUSSIAN INNOVATIONS) Empirical coverage levels of confidence intervals (nominal confidence level 90\%) constructed via four different methods (with the simple block size choice $b \sim n^{1 / 4}$ for the bootstrap). The standard error of each of these empirical probabilities is $\mathbf{0 . 0 1 5}$ as each is based on 400 series.

\begin{tabular}{|c|c|c|c|c|c|c|c|c|c|}
\hline$n$ & \multicolumn{3}{|c|}{60} & \multicolumn{3}{c|}{120} & \multicolumn{3}{c|}{240} \\
\hline$\rho$ & 0.6 & 0.9 & 1.0 & 0.6 & 0.9 & 1.0 & 0.6 & 0.9 & 1.0 \\
\hline CLT & 0.7775 & 0.7075 & 0.7750 & 0.7000 & 0.6550 & 0.7000 & 0.6775 & 0.6400 & 0.6600 \\
\hline CLT $-\eta$ & 0.7750 & 0.7150 & 0.7750 & 0.7125 & 0.6575 & 0.7075 & 0.6850 & 0.6325 & 0.6550 \\
\hline BBB & 0.8750 & 0.8050 & 0.8175 & 0.8575 & 0.7600 & 0.7825 & 0.8250 & 0.7675 & 0.7925 \\
\hline BBB- $\eta$ & 0.8825 & 0.8200 & 0.8275 & 0.8400 & 0.7725 & 0.7900 & 0.8250 & 0.7775 & 0.8050 \\
\hline
\end{tabular}

Table 2: (ARCH INNOVATIONS) Empirical coverage levels of confidence intervals (nominal confidence level 90\%) constructed via four different methods (with the simple block size choice $b \sim n^{1 / 4}$ for the bootstrap). The standard error of each of these empirical probabilities is 0.015 as each is based on 400 series. 
From Table 1 it is apparent that all four methods appear to work well in the Gaussian case, with the CLT methods outperforming the BBB methods in terms of achieving (or coming close to achieving) the nominal coverage level of $90 \%$. This phenomenon may be attributed to the choice $b \sim n^{1 / 4}$ being suboptimal, as there is no reason to expect that $c_{X}=1$ in eq. (16).

As expected, Table 2 shows that the CLT and CLT $-\eta$ methods do not work well in the case of non-i.i.d. (ARCH) innovations in which case the blocks-of-blocks bootstrap definitely outperforms the CLT methods.

Interestingly, the gain in performance from using the transformation $\eta$ in connection with the CLT and/or the BBB does not appear to be significant; this is partly explainable by viewing a plot of the $\eta(x)$ function, and noting that it is almost linear for $x$ in the interval $(0.5,1)$. Similarly, the $\lambda(x)$ function that is suggested in the case of possible negative unit root is almost linear for $x$ in the interval $(-1,-0.5)$.

Note that the simple block size choice $b \sim n^{1 / 4}$ gives reasonable (albeit not perfect) results as Tables 1 and 2 demonstrate. To be more precise, it seems that this common (for the two cases) block size choice $b \sim n^{1 / 4}$ is closer to being optimal in the Gaussian case as compared to the case of ARCH innovations.

We now address the question of actually finding the optimal block size, i.e., empirically pin-pointing the unknown constant $c_{X}$ for the two different time series models considered above.

To do this, a simulation experiment was carried out in which a number of stretches $\left\{X_{1}, \ldots, X_{n}\right\}$, with $n=240$, were generated from model (1) with $\mu=0$, and five different values for $\rho=0.6,0.7,0.8,0.9$ and 1 . As before, we used the same two choices for the innovation sequence $\left\{\epsilon_{t}\right\}$ (Gaussian and ARCH).

We used the block size specification $b=\left[c n^{1 / 4}+0.1\right]$, and considered (and compared) different choices for the constant $c$ in the range $(1 / 4,4)$. The $c$-choice yielding a coverage level closest to the nominal $90 \%$ formed our empirical estimate $\hat{c}_{X}$ of $c_{X}$. Our results are summarized in Figure 1 below giving plots of $\hat{c}_{X}$ vs. $\rho$ in the two cases considered (Gaussian and ARCH innovations). 
Note that the jaggedness in the plots of Figure 1 is due to the Monte Carlo error in estimating this optimal constant $c_{X}$; this error can be reduced by conducting an experiment of a bigger scale but this is currently not feasible and/or cost-effective: suffices to note that the simulation leading to Figure 1 took almost three days to run on a SUN Sparcstation.

Nevertheless, Figure 1 yields the following significant insights:

1. The optimal constant $c_{X}$ seems to depend on $\rho$ as expected. However, after smoothing the plot of $\hat{c}_{X}$ vs. $\rho$, (e.g., by fitting a straight line), it seems that $c_{X}$ may well be a decreasing function of $\rho$ which is surprising.

2. The optimal constant $c_{X}$ definitely depends on probabilistic features of the $\left\{X_{t}\right\}$ series that go beyond the value of $\rho$; this is apparent from the fact that $c_{X}$ seems larger (maybe even double) in the Gaussian innovations case as compared to our (particular) ARCH innovations case.

3. Last but not least is an observation that is not directly manifest in Figure 1 (which is only a summary of our simulation), but is obvious from an examination of the totality of our simulation results; namely that: (a) using the optimal constant $c_{X}$ manages to yield the desired coverage level equal (or very close) to the nominal, and that (b) the achieved coverage levels remain close to the nominal even under some misspecification of the constant $c$ used, e.g., using a constant $c$ three of four times larger than the optimal $c_{X}$. 


\section{Conclusions}

We have presented two new avenues for constructing confidence intervals for the unknown autoregressive root in the case of time series data $\left\{X_{1}, \ldots, X_{n}\right\}$ arising from the familiar AR(1) model (1); one avenue is based on the Central Limit Theorems of our Section 2, and the other on the blocks-of-blocks bootstrap of Section 3.

The two new methodologies rely on clever pre-processing (e.g. differencing) of the original series, and are free of the difficulties present in previous methods that were due to data nonstationarity and/or discontinuity in the limit distribution in the case of a unit root. We have also indicated normalizing and variance stabiling transformation that are applicable for both methodologies.

The proposed confidence intervals based on the first method (Central Limit Theorem) are very quick-and-easy to construct but are valid only under the assumption that the innovation sequence $\left\{\epsilon_{t}\right\}$ is i.i.d. By contrast, confidence intervals based on the second method (blocks-of-blocks bootstrap) do not require i.i.d. innovations for their validity; they do however require that the practitioner makes a reasonable choice for the block size, and they are computer-intensive as all bootstrap methods are.

Some finite-sample simulations were also presented supporting the practical applicability of the two proposed methods, and giving some insights on the issue of block size choice for the blocks-of-blocks bootstrap. 


\section{Appendix: Technical Proofs}

Proof of Lemma 1. The proof is immediate by taking cases.

If $-1<\rho<1$, then $\left\{Y_{t}\right\}$ is strictly (and weakly) stationary as the first-order difference of the strictly (and weakly) stationary sequence $\left\{X_{t}\right\}$. To compute $\operatorname{Corr}\left(Y_{t}, Y_{t+1}\right)$ in this case recall that $E X_{t}=\mu /(1-\rho)$, and $\operatorname{Cov}\left(X_{t}, X_{t+h}\right)=\rho^{|h|} \sigma^{2} /\left(1-\rho^{2}\right)$; cf. Brockwell and Davis (1991). Note that

$$
Y_{t}=\mu+\rho X_{t-1}+\epsilon_{t}-X_{t-1}
$$

and consequently

$$
E Y_{t}=\mu+(\rho-1) E X_{t-1}=0
$$

If we denote $m \equiv \mu /(1-\rho)$, then we have:

$$
\begin{gathered}
\operatorname{Var} Y_{t}=E\left(X_{t}-X_{t-1}\right)\left(X_{t}-X_{t-1}\right)=E\left(X_{t}-m+m-X_{t-1}\right)\left(X_{t}-m+m-X_{t-1}\right)= \\
=2\left(\operatorname{Var} X_{t}-\operatorname{Cov}\left(X_{t}, X_{t-1}\right)\right)=2(1-\rho) \sigma^{2} /\left(1-\rho^{2}\right) .
\end{gathered}
$$

Similarly,

$\operatorname{Cov}\left(Y_{t}, Y_{t+1}\right)=E\left(X_{t}-X_{t-1}\right)\left(X_{t+1}-X_{t}\right)=E\left(X_{t}-m+m-X_{t-1}\right)\left(X_{t+1}-m+m-X_{t}\right)=$

$=\operatorname{Cov}\left(X_{t}, X_{t+1}\right)-\operatorname{Var} X_{t}-\operatorname{Cov}\left(X_{t-1}, X_{t+1}\right)+\operatorname{Cov}\left(X_{t}, X_{t+1}\right)=\left(2 \rho-1-\rho^{2}\right) \sigma^{2} /\left(1-\rho^{2}\right)$, and the fact that $\operatorname{Corr}\left(Y_{t}, Y_{t+1}\right)=(\rho-1) / 2$ follows.

Now if $\rho=1$, then $Y_{t}=\mu+\epsilon_{t}$ which is (by assumption) strictly (and weakly) stationary. Also by assumption the sequence $\left\{\epsilon_{t}\right\}$ is uncorrelated, thus $\operatorname{Corr}\left(Y_{t}, Y_{t+1}\right)=0$. Since $(\rho-1) / 2=0$ in this case, it follows that $\operatorname{Corr}\left(Y_{t}, Y_{t+1}\right)=(\rho-1) / 2$ and the Lemma is proven.

Proof of Lemma 2. The case where $\rho=1$ is obvious. Now if $-1<\rho<1$, we have

$$
X_{t}=m+\sum_{k=0}^{\infty} \rho^{k} \epsilon_{t-k}=m+\epsilon_{t}+\rho \epsilon_{t-1}+\rho^{2} \epsilon_{t-2}+\cdots
$$


where this infinite sum of random variables converges almost surely and in mean-square - see e.g. Brockwell and Davis (1991). The above equation combined with the fact that $Y_{t}=\mu+(\rho-1) X_{t-1}+\epsilon_{t}$ proves the lemma.

Proof of Theorem 1. First note that asymptotic normality for $\hat{\rho}_{Y}$ ensues from the linear representation for $Y_{t}$ given in our Lemma 2 in conjunction with Theorem 7.2.2 in Brockwell and Davis (1991). The asymptotic variance $\tau_{Y}^{2}$ is given by Bartlett's formula:

$$
\tau_{Y}^{2}=\sum_{k=-\infty}^{\infty}\left(\rho_{Y}^{2}(k)+\rho_{Y}(k-1) \rho_{Y}(k+1)+2 \rho_{Y}^{2}(1) \rho_{Y}^{2}(k)-4 \rho_{Y}(1) \rho_{Y}(k) \rho_{Y}(k+1)\right),
$$

where $\rho_{Y}(k) \equiv \operatorname{Corr}\left(Y_{t}, Y_{t+k}\right)$; in this notation, $\rho_{Y}(1) \equiv \rho_{Y}$.

Note that by Theorem 3.2.1 in Brockwell and Davis (1991)) we have that:

$$
\rho_{Y}(k)=\frac{\sum_{j=0}^{\infty} \psi_{j} \psi_{j+|k|}}{\sum_{j=0}^{\infty} \psi_{j}^{2}}
$$

where $\psi_{0}=1$ and $\psi_{j}=(\rho-1) \rho^{j-1}$ by Lemma 2 .

A quick calculation shows that $\sum_{j=0}^{\infty} \psi_{j}^{2}=2 /(1+\rho)$, and $\sum_{j=0}^{\infty} \psi_{j} \psi_{j+|k|}=(\rho-$ 1) $\rho^{k-1} /(1+\rho)$, for $k>0$; it follows that

$$
\rho_{Y}(k)=(\rho-1) \rho^{k-1} / 2 \quad \text { for } \quad k>0 .
$$

We can also compute the following:

$$
\begin{gathered}
\sum_{k=-\infty}^{\infty} \rho_{Y}^{2}(k)=\frac{(\rho+3)}{2(\rho+1)}, \\
\sum_{k=-\infty}^{\infty} \rho_{Y}(k) \rho_{Y}(k+1)=\frac{(\rho-1)(\rho+2)}{2(\rho+1)},
\end{gathered}
$$

and

$$
\sum_{k=-\infty}^{\infty} \rho_{Y}(k-1) \rho_{Y}(k+1)=\rho(\rho-1)-\frac{(\rho-1)^{2}}{4}-\frac{(\rho-1)}{2(\rho+1)}
$$

after some easy algebra the result $\tau_{Y}^{2}=(\rho+1) / 2$ follows.

Proof of Theorem 2. Again, asymptotic normality for $\hat{\rho}_{W}$ ensues from the linear representation for $W_{t}$ given in our Lemma 4 in conjunction with Theorem 7.2.2 in 
Brockwell and Davis (1991). The asymptotic variance $\tau_{W}^{2}$ is again given by Bartlett's formula:

$\tau_{W}^{2}=\sum_{k=-\infty}^{\infty}\left(\rho_{W}^{2}(k)+\rho_{W}(k-1) \rho_{W}(k+1)+2 \rho_{W}^{2}(1) \rho_{W}^{2}(k)-4 \rho_{W}(1) \rho_{W}(k) \rho_{W}(k+1)\right)$,

where $\rho_{W}(k) \equiv \operatorname{Corr}\left(W_{t}, W_{t+k}\right)$; in this notation, $\rho_{W}(1) \equiv \rho_{W}$.

A calculation similar the one in the proof of Theorem 1 shows that

$$
\rho_{W}(k)=(\rho+1) \rho^{k-1} / 2 \quad \text { for } \quad k>0 .
$$

We can also compute the following:

$$
\begin{gathered}
\sum_{k=-\infty}^{\infty} \rho_{W}^{2}(k)=\frac{(\rho-3)}{2(\rho-1)}, \\
\sum_{k=-\infty}^{\infty} \rho_{W}(k) \rho_{W}(k+1)=\frac{(\rho+1)(\rho-2)}{2(\rho-1)}
\end{gathered}
$$

and

$$
\sum_{k=-\infty}^{\infty} \rho_{W}(k-1) \rho_{W}(k+1)=\rho(\rho+1)-\frac{(\rho+1)^{2}}{4}-\frac{(\rho+1)}{2(\rho-1)} ;
$$

after some easy algebra the result $\tau_{W}^{2}=(1-\rho) / 2$ follows.

\section{References}

[1] Andrews, D.W.K. (1984), Non-strong mixing autoregressive processes, Journal of Applied Probability 21, 930-934.

[2] Basawa, I.V., Mallik, A.K., McCormick W.P., Reeves, J.H., and Taylor R.L. (1991), Bootstrapping unstable first-order autoregressive processes. Annals of Statistics 19, 1098-1101.

[3] Bose, A. (1988), Edgeworth correction by bootstrap in autoregressions. Annals of Statistics 14, 1709-1722. 
[4] Brockwell, P.J. and Davis, R.A. (1991), Time Series: Theory and Models, 2nd edition. Springer-Verlag, New York.

[5] Datta, S. and Sriram, T.N. (1997), A modified bootstrap for autoregression without stationarity, Journal of Statistical Planning and Inference 59, 19-30.

[6] Dickey, D.A. and Fuller, W.A. (1979), Distribution of the estimators for autoregressive time series with a unit root. Journal of the American Statistical Association 74, 427-431.

[7] Davison, A.C. and Hinkley, D.V. (1997), Bootstrap methods and their application, Cambridge University Press, Cambridge UK.

[8] Doukhan, P. (1994), Mixing: Properties and Examples, Lecture Notes in Statistics \# 85, Springer, New York.

[9] Efron, B. and Tibshirani, R. (1986), Bootstrap methods for standard errors, confidence intervals, and other measures of statistical accuracy (with discussion), Statistical Science 1, 54-96.

[10] Efron, B. and Tibshirani, R. (1993), An introduction to the bootstrap, Chapman and Hall, New York.

[11] Elliot, G. and Stock, J.H. (1994), Inference in time series regression when the order of integration of a regressor is unknown. Econometric Theory 10, 672-700.

[12] Ferretti, N. and Romo, J. (1996), Unit root bootstrap tests for AR(1) models, Biometrika 83, 849-860.

[13] Freedman, D. (1984), On bootstrapping two-stage least-squares estimates in stationary linear models, Annals of Statistics 12, 827-842.

[14] Fuller, W. (1996), Introduction to Statistical Time Series, 2nd Ed., John Wiley, New York. 
[15] Hall, P. (1992), The Bootstrap and Edgeworth Expansion, Springer Verlag, New York.

[16] Hall, P., Horowitz, J.L, and Jing, B.-Y. (1996), On blocking rules for the bootstrap with dependent data, Biometrika 50, 561-574.

[17] Hamilton, J.D. (1994), Time Series Analysis, Princeton University Press, Princeton, New Jersey.

[18] Hansen, B.E. (1997), Bootstrapping the autoregressive model, manuscript (available via the internet: www2.bc.edu/ hansenb/).

[19] Künsch, H.R. (1989), The jackknife and the bootstrap for general stationary observations, Annals of Statistics 17, 1217-1241.

[20] Phillips, P.C.B. and Perron, P. (1988), Testing for a unit root in time series regression. Biometrika 75, 335-346.

[21] Politis, D.N. and Romano, J.P. (1992a), A General Resampling Scheme for Triangular Arrays of $\alpha$-mixing Random Variables with Application to the Problem of Spectral Density Estimation, Annals of Statistics 20, 1985-2007.

[22] Politis, D.N. and Romano, J.P. (1992b), A circular block-resampling procedure for stationary data, in Exploring the Limits of Bootstrap, (edited by Raoul LePage and Lynne Billard), John Wiley, pp. 263-270.

[23] Politis, D.N. and Romano, J.P. (1994a), The stationary bootstrap, Journal of the American Statistical Association 89, pp. 1303-1313.

[24] Politis, D.N. and Romano, J.P. (1994b), Large sample confidence regions based on subsamples under minimal assumptions, Annals of Statistics 22, pp. 20312050 .

[25] Politis, D.N., Romano, J.P. and Wolf, M. (1999), Subsampling, Springer Verlag, New York. 
[26] Priestley, M. B. (1981), Spectral Analysis and Time Series. Academic Press, New York.

[27] Radulovic, D. (1996), The bootstrap of the mean for strong mixing sequences under minimal conditions, Statistics and Probability Letters 28, 65-72.

[28] Romano, J.P. and Thombs, L.A. (1996), Inference for autocorrelations under weak assumptions, Journal of the American Statistical Association 91, 590-600.

[29] Romano, J.P. and Wolf, M. (1998), Subsampling confidence intervals for the autoregressive root, Technical Report, Dept. of Statistics, Stanford University.

[30] Shao, J. and Tu, D. (1995), The Jackknife and Bootstrap, Springer Verlag, New York.

[31] Stock, J.H. (1991), Confidence intervals for the largest autoregressive root in U.S. macroeconomic time series. Journal of Monetary Economics 28, 435-459. 